\title{
Análisis de la experiencia del Grupo de Teatro Inclusivo Rompecabezas desde las voces de sus protagonistas
}

Analysis of the experience of the Rompecabezas Inclusive Theater

Group from the voices of its protagonists

\author{
Volumen 21, Número 3 \\ Setiembre - Diciembre \\ pp. 1-35
}

\author{
Viviana González Rojas \\ Alicia Sandoval Poveda
}

Citar este documento según modelo APA

González Rojas, Viviana. y Sandoval Poveda, Alicia. (2021). Análisis de la experiencia del Grupo de Teatro Inclusivo Rompecabezas desde las voces de sus protagonistas. Revista Actualidades Investigativas en Educación, 21(3), 1-35. Doi. 10.15517/aie.v21i3.48156 


\title{
Análisis de la experiencia del Grupo de Teatro Inclusivo Rompecabezas desde las voces de sus protagonistas
}

Analysis of the experience of the Rompecabezas Inclusive Theater Group from the voices of its protagonists

\author{
Viviana González Rojas ${ }^{1}$ \\ Alicia Sandoval Poveda²
}

\begin{abstract}
Resumen: Este artículo relata la réplica de la experiencia de Loyd con el Grupo de Teatro Inclusivo Rompecabezas, con el objetivo de indagar la satisfacción, apreciación, recuerdos y aprendizajes de las personas participantes en el proyecto. El Grupo Rompecabezas forma parte de un proyecto de la Universidad Estatal a Distancia, que se desarrolla en San José, Costa Rica. De este se desprende la experiencia que da pie a este artículo desde un enfoque cualitativo y un alcance descriptivo. A finales de 2018 se aplicó una entrevista multimodal a 18 participantes del grupo con un rango de edad entre los 13 y 27 años, con Trastorno del Espectro Autista, Síndrome de Down y otras situaciones de discapacidad. Los resultados manifiestan un gusto generalizado de las personas participantes por el teatro y las diversas actividades relacionadas con el proyecto. Las opiniones de quienes participaron en relación con los beneficios que el teatro les aportaba, estuvieron enfocadas al trabajo con amigos, el triunfo colectivo y la diversión. Fue posible determinar que recuerdan y reconocen, en algunas ocasiones con apoyo, los espacios principales de las actividades del taller de teatro. La metodología aplicada para recuperar los puntos de vista de todas las personas participantes demostró ser accesible a todos sus estilos comunicativos. Se evidencian los aspectos que se proponían evaluar en esta experiencia en relación con el grado de satisfacción, los recuerdos, la apreciación y los aprendizajes de las personas participantes en el proyecto.
\end{abstract}

Palabras clave: teatro, igualdad de oportunidades, metodología, participación social.

Abstract: This article relates the replication of Loyd's experience with the Rompecabezas Inclusive Theater Group, with the objective of investigating the satisfaction, appreciation, memories and learnings of the participants in the project. The Rompecabezas Group is part of a project of the Universidad Estatal a Distancia, which is developed in San José, Costa Rica. It's from this project that rises the experience described in this article, from a qualitative approach and an exploratory-descriptive scope. At the end of 2018, a multimodal interview was applied to 18 participants of the group with an age range between 13 and 27 years old, with Autism Spectrum Disorder, Down Syndrome and other disability situations. The results show a generalized liking of the participants for the theater and the various activities related to the project. The opinions of the participants in relation to the benefits that the theater brought them were focused on working with friends, collective triumph and fun. It was possible to determine that they remember and recognize, sometimes with support, the main spaces of the theater workshop activities. The methodology applied to recover the points of view of all the participants proved to be accessible to all their communicative styles. It was possible to highlight the aspects that were intended to be evaluated in this experience in relation to the degree of satisfaction, memories, appreciation and learnings of the people participating in the project.

Key Words: theater, equal opportunities, methodology, social participation.

\footnotetext{
${ }_{1}$ Profesora de la Universidad Estatal a Distancia, de la Escuela Ciencias de la Educación, San José, Costa Rica. Licenciada en Educación Especial de la Universidad de Costa Rica. Dirección electrónica: vgonzalez@uned.ac.cr Orcid https://orcid.org/0000-0002-3114-4416

${ }^{2}$ Asistente de proyectos estratégicos de la Universidad Estatal a Distancia, en la Vicerrectoría de investigación, San José, Costa Rica. Licenciatura en Psicología de la Universidad de Costa Rica. Dirección electrónica: asandovalp@uned.ac.cr Orcid https://orcid.org/0000-0002-9678-7586
}

Artículo recibido: 18 de noviembre, 2020

Enviado a corrección: 24 de febrero, 2021

Aprobado: 16 de agosto, 2021 


\section{Introducción}

El proyecto "Desarrollo de habilidades sociales en jóvenes con Trastornos del Espectro Autista (TEA) a través de una experiencia artística: el teatro" de la Universidad Estatal a Distancia de Costa Rica, surge de la necesidad identificada por un grupo de madres de jóvenes con TEA de buscar espacios de esparcimiento, formación y socialización para quienes estén prontos a dejar el sistema educativo formal. Esta demanda recibe respuesta por parte de la UNED por medio de un proyecto de investigación-acción en el cual se realizan talleres de formación actoral con jóvenes en un ambiente real de teatro, con el objetivo de desarrollar y mejorar las habilidades sociales de estos. Del seno de este proyecto, nació el Grupo de Teatro Rompecabezas conformado por personas con y sin alguna situación de discapacidad, personas que requieren apoyos en el desarrollo de habilidades sociales y un equipo interdisciplinario.

El proyecto inició en 2016 en una alianza estratégica con el Teatro La Máscara, ubicado en San José, Costa Rica, con un primer grupo de formación actoral con jóvenes del Gran Área Metropolitana, y en el año 2018 se realizó otra convocatoria para iniciar un segundo grupo. Las clases fueron impartidas por una educadora que además es actriz profesional y profesora de actuación de la academia del Teatro La Máscara. Se realizaron seis montajes bajo la dirección de un reconocido director de teatro, cuatro con el primer grupo, uno con el segundo y uno con el grupo unificado, las cuales se han presentado ante el público al menos una vez cada una. El proyecto no tiene financiamiento de otras empresas, instituciones $u$ organizaciones, es un proyecto inscrito en la Vicerrectoría de investigación y el Centro de Investigaciones en Educación de la UNED, los cuales aportan un puesto de asistencia y materiales de papelería, impresiones, fotografía y materiales puntuales para los talleres. Gastos de vestuario y escenografía corren por parte de las familias y un porcentaje de lo generado por las entradas de las obras de teatro.

Cada sesión de trabajo tuvo una estructura similar, conformada por un calentamiento (estiramiento, movimiento corporal y música), montaje de escenas (aprendizaje del texto y la marcación de movimientos en escena) o el montaje de coreografías de baile. Además de las sesiones de teatro, con el primer grupo se realizaron sesiones de trabajo con elementos tecnológicos y la grabación ante cámaras para un documental. Ambos grupos han sido entrevistados por medios de divulgación masiva como la radio y la televisión. Como parte de ese proceso investigativo se ha llevado a cabo la recolección de información por medio de registro fotográfico, observaciones no participativas, bitácoras de sesiones realizadas por las 
personas facilitadoras, perfiles realizados por familias y docentes y grupos de discusión de familias y equipo de investigación. No obstante, desde el modelo social de la discapacidad, existía la necesidad de identificar una estrategia que permitiera recolectar la percepción de las personas participantes con respecto a su involucramiento en el grupo de teatro, independientemente de su capacidad de comunicación verbal.

En la experiencia recopilada en este artículo en particular con el grupo de participantes del Grupo Rompecabezas, se realizó una entrevista multimodal aplicada a cada participante del grupo en situación de discapacidad o que requerían apoyos para el desarrollo de habilidades sociales, para recopilar sus impresiones sobre el trabajo realizado en el proyecto. Para esto, fue necesario hacer una revisión de investigaciones previas donde se hubiera dado espacio a la voz de personas en situación de discapacidad dentro de procesos de investigación.

Las investigaciones en las que participan personas en situación de discapacidad, han estado caracterizadas tradicionalmente por estar a cargo únicamente de personas investigadoras profesionales, situando a sus protagonistas como meros espectadores, sin voz o representatividad alguna (Stack y McDonald, 2014). De hecho, existe un debate sobre cuánta voz se está dando en la investigación a las personas en situación de discapacidad y cómo solo se incluye la voz de aquellas que tienen habilidad verbal, lo que resulta ser poco equitativo cuando no se busca la forma de incluir en las investigaciones las experiencias de personas en situación de discapacidad, con dificultades de comunicación o que carecen de habilidad verbal (Hens, Robeyns y Schaubroeck, 2019). Lo anterior, no solo por los modelos de concepción de la discapacidad que se han manejado durante mucho tiempo y que siguen estando presentes, en los cuales la opinión y visión de las "personas expertas" son a las que se le asigna valor; sino también por los retos que plantea el desarrollo de investigaciones inclusivas, en especial por las posibilidades de comunicación de algunas personas en situación de discapacidad y la búsqueda de estrategias para la recolección y devolución de la información, más allá de los formatos del lenguaje oral o escrito.

Al respecto, varias han sido las experiencias e iniciativas de búsqueda de alternativas, metodologías y estrategias para incluir a las personas en situación de discapacidad, como participantes activos e informantes de cualquier proceso de investigación al que formen parte, las cuales funcionan como antecedentes metodológicos de la presente experiencia y se presentan a continuación. 
Preece y Jordan (2010) documentan el proceso desarrollado para obtener las opiniones de menores de edad con TEA que se comunicaban de forma verbal o mediante apoyos y sistemas aumentativos y alternativos de comunicación, sobre sus vivencias cotidianas, sus gustos, disgustos y sus deseos; así como su experiencia con los trabajadores sociales y sus servicios.

El proceso se realizó mediante entrevistas en las cuales se les brindó apoyos visuales individualizados y fotografías para que pudieran responder según sus necesidades de comunicación individual. Si bien se derivaron algunos retos en el proceso dadas las dificultades en la comunicación e interacción social propias de la condición de los y las participantes, las personas autoras resaltan el logro en la recolección de datos valiosos y relevantes, pero, sobre todo, el aporte al conocimiento existente al dar voz a las opiniones de menores de edad con TEA (Preece y Jordan, 2010).

Lewis (2009) aporta también acerca de un proceso desarrollado para escuchar de forma directa a niños y niñas con TEA sobre sus puntos de vista en relación con la espiritualidad y la religión. En relación con la metodología utilizada, se destaca como válido alentar a que comuniquen cuando no comprenden una pregunta o no saben la respuesta, así como a solicitar el replanteamiento de la pregunta cuando esto sucede, ya que conduce a mejoras en la precisión de las respuestas dadas. Se propone además el uso de oraciones en lugar de preguntas, preguntar en secuencia y el derecho a guardar silencio.

Otra de las experiencias es la desarrollada por Danker, Strnadová y Cumming (2017), quienes involucran a estudiantes con TEA en la investigación por medio de una técnica visual cualitativa denominada Técnica de Investigación de Foto-Elicitación, comúnmente utilizada con grupos vulnerables para facilitar, con apoyo de fotografías, respuestas más sustanciosas en las entrevistas. El beneficio más importante de esta técnica es la posibilidad que brinda a las personas en situación de discapacidad de que sus voces auténticas sean escuchadas. Resaltan además la accesibilidad dado que no requiere que lean o escriban, la sensación de empoderamiento que les brinda, así como el hecho de que les ayuda a disminuir la ansiedad y angustia que participar en entrevistas tradicionales les puede producir.

Harrington, Foster, Rodger y Ashburner (2014), en su estudio sobre las experiencias escolares convencionales de jóvenes con TEA y sus familias en Queensland, Australia, compartieron algunas de las estrategias que fueron adaptadas para incluir a las personas con TEA como informantes en sus investigaciones. Por ejemplo: enfocar las preguntas en el presente, incluir mayormente preguntas cerradas y sobre emociones básicas, así como 
consultar previamente a las familias sobre las necesidades particulares de socialización o comunicación de cada joven, y la mejor forma de abordarlas.

Harrington et al. (2014) concluyeron que a los y las jóvenes con TEA, si se les brinda la oportunidad, pueden aportar valiosos conocimientos sobre sus propias experiencias en los procesos de investigación. Finalmente, Fletcher-Watson et al. (2018), elaboraron un marco de referencia para la participación de las personas con TEA en proyectos de investigación relacionados con ellos mismos. Entre sus resultados, se identificaron cinco temas relevantes e ideales que deben ser considerados en las prácticas de investigación participativas: respeto, autenticidad, supuestos, infraestructura y empatía (Fletcher-Watson et al., 2018).

Propiamente en el trabajo relacionado con teatro y discapacidad, es más frecuente encontrar estudios donde se utilizan instrumentos de recolección de datos como pruebas o escalas estandarizadas (Beadle-Brown et al., 2018; Corbett et al., 2014, Corbett et al., 2016; Guli, Semrud-Clikeman, Lerner y Britton, 2013; Ioannou, Key, Muscatello, Klemencic y Corbett, 2020; Mehling, Tassé y Root, 2017; Neufeld, 2012), uso de medidas fisiológicas (Corbett, Blain, loannou y Balser, 2017) observación directa (Corbett et al., 2014; Guli et al., 2013; Kempe y Tissot, 2012; Kim et al., 2015; Neufeld, 2012) o entrevistas con informantes, ya sean cuidadores o docentes (Beadle-Brown et al., 2018; Calafat-Selma et al., 2016; Corbett et al., 2014; Guli et al., 2013; Heeks, 2015; Neufeld, 2012)

A continuación, se presentan estudios de teatro con personas en situación de discapacidad que han tomado en cuenta directamente su punto de vista de lo realizado. Blanco, Regueiro y González (2016) realizaron una entrevista grupal con las personas participantes del proyecto, además de la observación participante y la revisión bibliográfica. Por su parte, Mehling et al. (2017) realizaron una entrevista con participantes y sus cuidadores para la validación social de la metodología, además de una medida con un reporte de las familias y una observación. Asimismo, Trowsdale y Hayhow (2013) realizaron una escala Likert completada por docentes sobre el desarrollo de la creatividad de las personas participantes. Junto a esto, desarrollaron herramientas para recopilar información de las personas participantes como discusiones, el uso de emoticonos y la creación de álbumes personales con fotos seleccionadas. Finalmente, Kim y Boyns (2015) realizaron entrevistas con personas con TEA, sin situaciones de discapacidad, familiares y público de las obras de teatro, además de la observación estructurada de las sesiones.

Sin embargo, el antecedente más importante para la experiencia reportada en este artículo es el de Loyd (2013), quien se dedicó a obtener los puntos de vista de estudiantes con 
TEA en relación con su participación en las clases de teatro. Las formas de comunicación de las personas participantes eran diversas, algunos utilizaban lenguaje verbal, otros solo palabras ocasionales, sin embargo, en su mayoría eran comunicadores no verbales.

El tipo de entrevista utilizada por la autora se describe como multimodal, de forma que los y las participantes contaban con diferentes formas tanto para acceder a las preguntas como para responderlas, las cuales estaban enfocadas en conocer sobre lo que les gustaba y no les gustaba de la escuela, del teatro, así como los sentimientos en relación con las actividades y presentaciones que realizaban (Loyd, 2013).

Si bien la autora describe la experiencia como retadora, invita a dejar de lado las etiquetas y demuestra como el estudiantado con una condición de TEA, aún con dificultades importantes de comunicación, fueron capaces de expresar sus pensamientos, sentimientos, preferencias y reflexiones en torno a una actividad, en este caso, el teatro (Loyd, 2013).

Esta metodología fue utilizada de base para la experiencia presentada en el presente artículo para conocer el punto de vista de las personas participantes con TEA, utilizando diferentes apoyos como fotos y videos, lo que involucra a todo el grupo independientemente de su competencia verbal.

El trabajo que aquí se muestra con el análisis del Grupo de Teatro Inclusivo Rompecabezas, también es prueba de ello. Esta experiencia se llevó a cabo entre noviembre de 2018 y febrero de 2019, con el objetivo de averiguar el grado de satisfacción de las personas implicadas en el grupo, explorando sus recuerdos en el proceso, identificando su apreciación sobre las actividades en las que participaron y valorando los aprendizajes que los y las jóvenes adquirieron en su participación en el proyecto.

\section{Referente teórico}

EI TEA es un trastorno generalizado del neurodesarrollo que se caracteriza por alteraciones en dos grandes áreas de la persona: en la interacción social y la comunicación, y en su patrón de comportamientos, intereses y actividades (Ministerio de Sanidad Servicios Sociales e Igualdad, 2019). Si bien son características comunes y definitorias las que se comparten, al ser un espectro, las manifestaciones son diversas de una persona a otra. Por ejemplo, en el área de la interacción y la comunicación social, se pueden evidenciar acercamientos sociales inusuales o inadecuados, dificultades para ajustar el comportamiento en diversos contextos sociales; habilidades lingüísticas adecuadas o menores a la esperadas para su edad, con dificultades para utilizarlas en una interacción o en el contexto indicado, con 
ausencia de una mirada mutua adecuada, y dificultades para reconocer e interpretar el lenguaje no verbal en otros (Asociación Americana de Psiquiatría, 2013).

En lo que comprende los patrones de comportamientos, intereses y actividades, las personas con TEA suelen ser excesivamente inflexibles y con tendencia al seguimiento de rutinas, con intereses muy restringidos y fijos. Asimismo, algunas personas con esta condición presentan alteraciones en el procesamiento de los estímulos sensoriales del entorno, ya sea con hiper- o hiporeactividad (Asociación Americana de Psiquiatría, 2013).

Existen varias razones por las cuales las personas con TEA suelen ser excluidas de los procesos de investigación, entre ellas la diversidad que engloba este colectivo, desde personas con alguna discapacidad intelectual u otras condiciones asociadas, hasta aquellas con capacidades cognitivas incluso por encima del promedio, que no se identifican como sujetos con alguna situación de discapacidad (Dickins, 2008, Aitken y Millar, 2004 en Brewster y Coleyshaw, 2011). De acuerdo con Preece (2002, en Brewster y Coleyshaw, 2011) las dificultades en comunicación verbal y no verbal, así como en la interacción social, influyen en la motivación de la persona para comunicarse, en la comprensión de conceptos abstractos relacionados con tiempo y emociones, así como en la posibilidad de reflexionar sobre sus sentimientos y preferencias, por lo que suelen ser las principales justificaciones para la exclusión de esta población en los estudios vinculantes con ella misma.

Numerosos autores, como Pallisera, Fullana, Puyaltó, Vila y Díaz (2017) y Susinos y Parrilla (2013), reconocen que la participación en procesos de investigación de las personas en situación vulnerable, como aquellas con alguna situación de discapacidad, se ha caracterizado en general a través de los años por ser escasa, y porque su colaboración se ha dado desde un rol pasivo y espectador, como "sujetos investigados", sin realmente considerar sus criterios, perspectivas y en la toma de decisiones en la propia investigación o como sujetos de la misma investigación.

Si bien es un tema pendiente, en los últimos años se ha producido un creciente interés por involucrar y dotar de protagonismo a las personas en situación de discapacidad en los procesos de investigación (Pallisera, Puyaltó, Fullana, Vila y Martín, 2015), producto de la transformación progresiva de la concepción sobre la discapacidad, la implementación del modelo social fundamentado en los derechos humanos, así como la publicación de la Convención de Naciones Unidas sobre los Derechos de las personas con Discapacidad en el año 2006 (Organización de las Naciones Unidas, 2006). 
A lo largo del tiempo se ha concebido la discapacidad desde tres modelos, como resultado de las influencias sociopolíticas, culturales y económicas propias de cada época histórica. Inicialmente dominó el modelo de la prescindencia, el cual atribuía la discapacidad a motivos religiosos, por lo que las personas en esta situación eran dignas de caridad y asistencia. Posteriormente, desde el modelo rehabilitador, se consideraba como causa de la discapacidad las limitaciones individuales de las personas, persiguiéndose como tratamiento la rehabilitación para "normalizarlas" (Palacios, 2008).

Luego de una lucha de las propias personas en situación de discapacidad quienes reclamaban su autonomía y su derecho a decidir respecto de su propia vida, se llega al modelo vigente, el social, el cual considera que las causas que originan la discapacidad no son religiosas ni científicas, ni están en el individuo, sino en el contexto que los acoge o los rechaza (Palacios, 2008; Victoria, 2013).

El modelo social de la discapacidad nació sostenido de la filosofía de vida independiente, desde la premisa de que toda vida humana es igualmente digna, que las personas en situación de discapacidad tienen el derecho a ser tratadas con igual respeto que al resto de sus semejantes, que deben tener la posibilidad de tomar sus propias decisiones, que gozan del derecho a participar plenamente en todas las actividades de la vida en comunidad, y que son capaces de aportar a la comunidad en igual medida que el resto (Palacios, 2008).

Lo anterior viene a ser respaldado por la Convención sobre los derechos de las personas con discapacidad, la cual reconoce el derecho de este colectivo a vivir de forma independiente y a ser incluido en la comunidad, así como a participar de forma activa en los procesos de adopción de decisiones sobre políticas y programas, incluidos los que les afectan directamente (Organización de las Naciones Unidas, 2006), para lo cual se torna evidente la necesidad de un cambio significativo en la consideración del papel que las personas en situación de discapacidad juegan dentro de todos los procesos de evaluación e investigación vinculados con ellos mismos.

Durante las décadas de los 60 y 70 , el rol de la persona en situación de discapacidad dentro de los procesos investigativos fue el de ser observadas y sometidas a pruebas para ser descritas y analizadas, siendo el equipo investigador quien tenía el poder y controlaba el estudio (Walmsley, 2001 en Pallisera y Puyalto, 2014). A partir de las décadas de los 80 y 90, se identifica que la no participación de las personas en situación de discapacidad en los estudios no permitía que los resultados de estos respondieran a las necesidades reales de la población, lo que dio paso a estilos de investigación más inclusivos en los que se les 
consideraba para entrevistas o para la evaluación de servicios (Tuffrey-Wijne y Butler, 2009 en Pallisera y Puyalto, 2014).

Para el final de la década de los 90, el debate avanzó hacia cuál era el enfoque idóneo para el rol de la persona en situación de discapacidad dentro de las investigaciones, si el de una participación activa, o una de mayor control como parte de un proceso de empoderamiento (Dowse, 2009, Young y Chesson, 2007 en Pallisera y Puyalto 2014). Es hasta los inicios del año 2000, que aumenta el número de estudios desarrollados desde un enfoque de colaboración más dinámico, en el cual la persona en situación de discapacidad participa no solo como asesora, sino como coinvestigadora o investigadora principal desarrollando proyectos propios (Walmsley y Johnson, 2003, Ward y Simons, 1998, en Pallisera y Puyalto, 2014).

Estas personas son los propios expertos de sus vidas y así como lo indican Abbott y McConkey (2006) en Fullana, Pallisera, Vilá y Puyaltó, (2016):

La voz de las personas con discapacidad es fundamental para conocer qué es lo que ellas desean en sus vidas, cómo valoran los servicios que se les ofrecen y para poder planificar y desarrollar los apoyos necesarios que garanticen su participación en todos los ámbitos de la comunidad. (p. 113)

Lo anterior se ha defendido bajo el argumento que cuando no hay presencia de personas en situación de discapacidad en investigación, esta es percibida por el mismo colectivo como una violación de su experiencia, una práctica irrelevante pues no se reconoce su voz ni se contempla sus necesidades, lo que no permite, por lo tanto, la mejora real de su calidad de vida (Oliver, 1992 en Susinos y Parrilla, 2013).

La comunidad científica se ha visto ante el reto de transgredir las fronteras tradicionales, de aumentar los espacios de acción, y de implementar prácticas que contribuyan a la generalización de un enfoque verdaderamente inclusivo dentro de la investigación, entre ellas: dejar atrás la tradición académica del análisis de la discapacidad desde una única disciplina, favorecer espacios de diálogo colectivos con actores que habitualmente no han sido tomados en consideración, y una reflexión más amplia sobre la pertinencia y adecuación de los enfoques metodológicos utilizados para la construcción del conocimiento, con el objetivo de poder dar voz y conseguir una presencia más real y activa de las personas protagonistas que los métodos tradicionales les ha negado por tanto tiempo (Susinos y Parrilla, 2013). 
También es necesario hacer esto en las investigaciones sobre teatro y discapacidad, donde tradicionalmente las personas en situación de discapacidad han sido receptoras del proceso, observadas y evaluadas por sus progenitores, cuidadores e investigadores, y tan solo en algunas ocasiones se les ha preguntado directamente, a través de alguna metodología, su opinión al respecto (Blanco et al., 2016; Kim y Boyns, 2015; Mehling et al., 2017; Trowsdale y Hayhow, 2013).

El teatro como herramienta para mejorar las habilidades sociales en población en situación de discapacidad, en especial con TEA, se ha venido trabajando desde hace varias décadas (Sherratt y Peter, 2002). Se ha planteado la importancia de realizar el abordaje desde las habilidades e intereses de las personas participantes con estructuras narrativas flexibles con una improvisación guiada, desde obras de teatro participativas se trabaja la imaginación, la emoción y la empatía contribuyendo en el desarrollo social, mejorando la comunicación verbal y física, la interacción social, imaginación social y creatividad (Shaughnessy, 2016).

La integración del juego y la actuación en el trabajo desde diferentes programas con personas con TEA aumenta su compromiso en actividades con otros y con sus propias necesidades, les permite experimentar las reglas de la socialización, los roles y la autorregulación (Smagorinsky, 2016). También se ha trabajado desde el teatro para la mejora de habilidades sociales con personas con otras situaciones de discapacidad además del TEA, como grupos con discapacidades intelectuales (García, 2014; Thergaonkar y Daniel, 2019; Zyga, Russ, Meeker y Kirk, 2017), Síndrome de Down (González, 2017; González, 2018; Toledo, 2020) necesidades educativas múltiples (Narváez, Parra y Sepúlveda, 2020), personas con condiciones de salud mental (Fernández, Guerra y Begara, 2013) y grupos inclusivos en los que participan personas con situaciones de discapacidad y sin estas (Bustamante, Miranda y Sánchez, 2020; Cook, 2020; Lenekakis y Koltsida, 2017). Sin embargo, en estos planteamientos se trabaja desde el interés de la persona en situación de discapacidad, pero no se le integra en el planeamiento o la evaluación de la metodología empleada.

Además, más allá de las intervenciones terapéuticas o educativas, el teatro es un arte y como tal, las personas tienen derecho a participar de él, aunque en muchas ocasiones se les prive de los espacios para desarrollar sus talentos e intereses artísticos o para ser reconocidos como artistas (Vargas-Pineda y López-Hernández, 2020). El espacio abierto en el Grupo Rompecabezas les permite acceso tanto a la formación en teatro como a ser reconocidos como actores y actrices en sus presentaciones. Por tanto, este trabajo permite explorar la 
percepción de participantes de un grupo artístico donde se busca la mejora de las habilidades sociales, pero también el acercamiento al arte.

\section{Metodología}

\subsection{Enfoque}

El Grupo de Teatro Rompecabezas nació como una compañía de teatro dentro del proyecto de investigación acción de la UNED "Desarrollo de habilidades sociales a través de una experiencia artística: el teatro", conformado por personas con y sin diferentes situaciones de discapacidad y que se encuentra en funcionamiento desde el año 2016.

En el presente artículo se presenta una investigación realizada con las personas en situación de discapacidad del Grupo de Teatro Rompecabezas con el objetivo de indagar su satisfacción con el trabajo en el grupo, explorar sus recuerdos del proceso, identificar su apreciación sobre las actividades en las que participaron y valorar los aprendizajes que adquirieron sobre las actividades realizadas en el teatro. Además, se pretende visibilizar el uso de una metodología que permite incluir la voz de todas las personas participantes en este tipo procesos. En esta experiencia en particular se trabajó desde un enfoque cualitativo, recogiendo por medio de una entrevista multimodal las impresiones de las personas participantes del grupo. El estudio tiene un alcance descriptivo dado su novedad y falta de antecedentes en el país, y su interés en describir la perspectiva de las personas participantes sobre la experiencia.

Debe destacarse que, en la situación particular del Grupo de Teatro Rompecabezas, existía en las personas participantes diversas formas de comunicación verbal oral así como formas alternativas, por ejemplo: aquellos que utilizaban señas, gestos naturales y palabras monosílabas para expresarse; o jóvenes con correcta articulación pero que requerían apoyos en el componente pragmático del lenguaje. Por esta razón, era necesario recurrir a una metodología que permitiera indagar y presentar las opiniones de todos y todas sin necesidad de una interpretación, independientemente de su forma de comunicación.

Para ello, se utilizó la entrevista multimodal de Loyd (2013) que presenta diferentes canales de comunicación (escrita, fotográfica y en video) para realizar la recolección de información. Se crearon categorías de respuesta según las preguntas realizadas y se analizó con qué frecuencia y qué porcentaje de las personas participantes dieron respuestas dentro de cada categoría. De esta manera, se utilizan frecuencias y porcentajes en el análisis de resultados para describir las respuestas obtenidas, en lugar de extraer frases y oraciones de 
respuestas específicas o interpretar la comunicación no verbal, pues esto priorizaría la voz de las personas con mayor habilidad verbal sobre la de los demás. No se utilizan cifras con la intención de realizar ningún tipo de inferencia o cálculo estadístico.

\subsection{Participantes}

El grupo de participantes en esta experiencia fueron 18 jóvenes con diferentes situaciones de discapacidad, 10 hombres y 8 mujeres, con una media de edad de 19,33 años $(\mathrm{DE}=4,17)$. Se entrevistó a todos los participantes activos del Grupo de Teatro Rompecabezas con una situación de discapacidad. Los criterios de inclusión para ser parte de la entrevista fueron ser personas con alguna situación de discapacidad o que requirieran apoyos en sus habilidades sociales, una capacidad básica de seguimiento de instrucciones y haber participado en el grupo durante el año 2018. Como criterio de exclusión se definió ser parte del Grupo Rompecabezas pero ser una persona sin una situación de discapacidad. Dentro del grupo entrevistado, se contó con 13 personas con TEA, 2 con Síndrome de Down, 1 con discapacidad cognitiva y 2 sin una situación de discapacidad pero que requerían apoyos en sus habilidades sociales.

En el análisis de datos que se presenta en este artículo se consideran las respuestas de las 18 personas participantes para todos los resultados excepto en dos casos. En primer lugar, en algunos momentos de la presentación de resultados se destacan las respuestas de las personas con TEA si el resultado obtenido se diferencia de los esperados desde la caracterización habitual de las personas con este diagnóstico. En segundo lugar, en las preguntas referentes a las obras de teatro en las que participaron y actividades de los primeros dos años del proyecto, el grupo se divide. El primer subgrupo está compuesto por 6 personas que ingresaron al proyecto en el año 2016 y habían participado de cuatro obras de teatro al momento de la realización de las entrevistas. El segundo, por 12 personas que ingresaron al proyecto en 2018 y habían participado de una sola obra de teatro para el momento de las entrevistas. En la tabla 1 se presenta el desglose de las personas participantes según género y año de ingreso al proyecto, así como el total de participantes. 
Tabla 1

Distribución por sexo de participantes del proyecto de teatro según grupo de participación y total de participantes del Grupo Rompecabezas en el año 2018

\begin{tabular}{|c|c|c|c|c|}
\hline \multicolumn{2}{|c|}{$\begin{array}{l}\text { Grupo } 1^{*} \\
\text { Tres años de participar del } \\
\text { proyecto }\end{array}$} & \multicolumn{2}{|c|}{$\begin{array}{c}\text { Grupo } 2^{* *} \\
\text { Un año de participar en el } \\
\text { proyecto }\end{array}$} & $\begin{array}{l}\text { Total de } \\
\text { participantes en el } \\
\text { proyecto }\end{array}$ \\
\hline Hombres & 4 & Hombres & 6 & 10 \\
\hline Mujeres & 2 & Mujeres & 6 & 8 \\
\hline Total & 6 & Total & 12 & 18 \\
\hline Media de edad & $19,67(\mathrm{DE}=5,28)$ & Media de edad & $19,17(\mathrm{DE}=3,76)$ & $\begin{array}{c}19,33 \text { años } \\
(\mathrm{DE}=4,17)\end{array}$ \\
\hline
\end{tabular}

*Ingreso al grupo en 2016

**Ingreso al grupo en 2018

Fuente: Elaboración propia, información de entrevistas realizadas a participantes del proyecto entre noviembre 2018- febrero 2019.

Se incluyen todas las respuestas de quienes cumplían los criterios de inclusión, independientemente de que dos de las personas participantes abandonaran el proyecto posteriormente, dado que su voz también se considera igualmente importante como parte del proceso. Sin embargo, en algunos momentos de la presentación de resultados, en caso de ser relevante, se señala si la respuesta fue de alguien que se retiró del proyecto posteriormente.

\subsection{Técnicas de recolección de información}

Se aplicó una entrevista multimodal basada en la utilizada por Doris Loyd (2013), que consiste en una entrevista en la cual las personas pueden acceder a las preguntas y dar respuesta a las mismas por diferentes medios: oral, escrito y visual. Esta propuesta ha sido valorada y tomada en consideración por diferentes autores (Cascio, Weiss y Racine, 2021; Danker, Strnadová y Cumming, 2019; Fayette y Bond, 2018; Tesfaye et al, 2019).

Este tipo de entrevista es recomendada por la autora para trabajar con personas con TEA o alguna otra situación de discapacidad porque, independientemente de las facilidades de comunicación y comprensión de la persona, permite disminuir la ansiedad y aumentar la accesibilidad, al brindar más claridad sobre las preguntas y las respuestas.

El instrumento de entrevista está conformado por tres partes:

1. Frases incompletas: esta primera parte de la entrevista está compuesta por ocho preguntas, cada una con tres opciones de respuesta. Estas exploran el gusto por el teatro, el gusto por las actividades que se realizan en el teatro, qué pueden hacer en las clases y en la presentación, y cómo se sienten en la clase y en la presentación. Para su aplicación se ponía sobre la mesa una tarjeta con la frase incompleta, tres tarjetas con cada una de las opciones de respuesta y al lado de estas, una fotografía alusiva para que la persona eligiera la respuesta. Las preguntas se encuentran en la tabla 2. 
Tabla 2

Preguntas y opciones de respuesta en la sección de frases incompletas de la entrevista aplicada a participantes del Grupo de Teatro Rompecabezas entre noviembre de 2018 y febrero de 2019

\begin{tabular}{|c|c|}
\hline Preguntas & Opciones de respuesta \\
\hline 1. Me gusta & $\begin{array}{ll}\text { a. } & \text { Teatro } \\
\text { b. } & \text { Cocinar } \\
\text { c. } & \text { Deporte }\end{array}$ \\
\hline 2. No me gusta & $\begin{array}{ll}\text { a. } & \text { Teatro } \\
\text { b. } & \text { Cocinar } \\
\text { c. } & \text { Deporte }\end{array}$ \\
\hline 3. En el teatro, me gusta & $\begin{array}{l}\text { a. Calentamiento } \\
\text { b. Baile } \\
\text { c. Practicar escenas }\end{array}$ \\
\hline 4. En el teatro, no me gusta & $\begin{array}{l}\text { a. Calentamiento } \\
\text { b. Baile } \\
\text { c. Practicar escenas }\end{array}$ \\
\hline 5. El teatro me ayuda a & $\begin{array}{l}\text { a. Trabajar con amigos } \\
\text { b. Ser valiente } \\
\text { c. Divertirme }\end{array}$ \\
\hline 6. En la presentación puedo & $\begin{array}{l}\text { a. Triunfar con mis compañeros } \\
\text { b. Hacer reír a la gente } \\
\text { c. Presentarme ante otras personas }\end{array}$ \\
\hline 7. En el teatro me siento & $\begin{array}{l}\text { a. Feliz } \\
\text { b. Nervioso } \\
\text { c. Triste }\end{array}$ \\
\hline 8. En la presentación me siento & $\begin{array}{ll}\text { a. } & \text { Feliz } \\
\text { b. Nervioso } \\
\text { c. }\end{array}$ \\
\hline
\end{tabular}

Fuente: Elaboración propia. Adaptado de Loyd (2013).

2. Fotografías: la segunda parte de la entrevista presenta una serie de fotografías divididas en dos grupos, como se esquematiza en la tabla 3

a. De actividades: se presentan tres grupos compuestos por tres fotografías cada uno. Los grupos de fotos corresponden a (1) actividades con el grupo (clase, recesos de la clase y celebraciones de cumpleaños), (2) actividades en las clases (calentamiento, montaje de escenas y baile) y (3) actividades fuera del teatro (sesiones de tecnología, grabación de documental y atender a la prensa). Sobre las sesiones de tecnología y la grabación del documental se preguntó solo a las seis personas que ingresaron en 2016, pues los demás no participaron en estas actividades. Para su aplicación, se presenta a la persona dos fotografías de una persona haciendo el signo de "me gusta" y de "no me gusta". Una a una se le presentan las fotografías de cada bloque para que describa qué sucede en ella y elija si le gusta o no la actividad. Si no recordaba qué estaba sucediendo en la 
fotografía, se le brindaban apoyos para rememorar, como por ejemplo "¿qué estabas haciendo ahí?” o “¿estaban hablando o bailando?”. Posteriormente, se le presentan juntas las fotografías de las tres opciones de cada grupo (1, 2 y 3), para que escoja cuál le gusta más y cuál menos.

b. De las obras de teatro: se les presenta una fotografía de cada una de las obras de teatro en las que aparecieron. Se les pide por cada una, describir qué sucedía en la obra y cuál era su personaje. Si no lo recordaban, se brindaban apoyos del mismo tipo que en la sección anterior.

Tabla 3

Clasificación de las actividades ilustradas en las fotografías de los tres primeros grupos de fotografías en la segunda parte de la entrevista aplicada a participantes del Grupo de Teatro Rompecabezas entre noviembre de 2018 y febrero de 2019

\begin{tabular}{|c|c|c|}
\hline $\begin{array}{l}\text { Tipo de } \\
\text { actividad }\end{array}$ & Actividades & Fotografías \\
\hline \multirow[t]{3}{*}{ Actividades } & 1. Actividades con el grupo & $\begin{array}{l}\text { a. Ensayo/Clase } \\
\text { b. Hablar con otros entre clases } \\
\text { c. Actividades sociales }\end{array}$ \\
\hline & $\begin{array}{l}\text { 2. Actividades durante las } \\
\text { clases }\end{array}$ & $\begin{array}{l}\text { a. Calentamiento } \\
\text { b. Baile } \\
\text { c. Practicar escenas }\end{array}$ \\
\hline & $\begin{array}{l}\text { 3. Actividades fuera de } \\
\text { clase }\end{array}$ & $\begin{array}{l}\text { a. Tecnología } \\
\text { b. Prensa y divulgación } \\
\text { c. Grabación de las obras para documental }\end{array}$ \\
\hline Obras de teatro & 4. Obras de teatro & $\begin{array}{l}\text { a. ¿De qué se trata la obra? } \\
\text { b. ¿Qué hacías en esta obra? }\end{array}$ \\
\hline
\end{tabular}

Fuente: Elaboración propia, basado en el formato de entrevistas realizadas entre noviembre 2018febrero 2019.

3. Videos: en la tercera parte de la entrevista se presenta, en una computadora o un dispositivo móvil, un video del grupo realizando una actividad de calentamiento y ejercicios actorales. Se le pide a la persona que describa qué estaba sucediendo, cómo se sentía en esa actividad y si le gusta o no realizarla. En el caso del primer grupo, se les presentaba también el video de una de sus obras de teatro donde se le preguntaba qué estaba haciendo, cómo se sentía y qué estaba haciendo el personaje en ese momento. Al segundo grupo no fue posible hacerle esa pregunta porque no se contaba con una grabación de su obra todavía.

En todas las secciones se brindaron apoyos a las personas participantes cuando tenían dificultad para dar una respuesta. Los apoyos utilizados fueron: 
- Cuando no recordaban con la fotografía o el video de una obra o personaje, recordarle aspectos generales del contexto sobre el que se consultaba: por ejemplo, si no recordaban la obra se le daban opciones "¿era la obra sobre navidad o sobre la naturaleza?"

- Cuando les costaba elegir entre tres opciones de respuesta, se les ponía a elegir entre ellas en grupos de dos. Primero entre 1 y 2 , luego entre 2 y 3 , luego entre 3 y 1 , y si no quedaba claro todavía, entre las dos que hubiera escogido más veces.

- Cuando tenían dudas de decir que algo no les gustaba, reasegurarles que no hay problema en que algo no le guste.

- Cuando había dificultad para dar una respuesta al elegir entre varias preguntas, se replanteaba con el mismo sentido. Por ejemplo, si no podían decidir cuál era su actividad favorita, se planteaba "si solo hubiera tiempo de hacer una de las tres actividades, ¿cuál escogerías?".

Cada una de las entrevistas se realizó de manera individual, en una sala privada, con dos facilitadoras. A cada participante se le realizó un encuadre de lo que se iba a trabajar en la sesión de entrevista y se le brindaron instrucciones específicas para cada parte. Una educadora especial realizó todas las preguntas y presentaba las opciones a la persona participantes, y una psicóloga realizaba la observación de la entrevista e iba facilitando en orden a su compañera los diferentes materiales.

Cada sesión se realizó en la lengua materna de la persona entrevistada (una en inglés y el resto en español), se llenó por cada una un protocolo de observación y registro de las respuestas y en el caso de aquellas personas que estuvieron de acuerdo, se grabó en video. Se contaba con consentimiento informado para estas entrevistas, dado que el documento de consentimiento para la participación del proyecto del Grupo Rompecabezas incluye la realización de entrevistas y otros métodos de evaluación con las personas participantes, así como el uso de fotografías, imágenes y videos para fines académicos.

\subsection{Análisis de la información}

Se construyeron dos grandes categorías de análisis con base en los objetivos de esta indagación: la primera, apreciación y satisfacción con los diferentes aspectos del proceso y la segunda, recuerdos y aprendizajes de las actividades realizadas. Posteriormente, se clasificaron las respuestas de las diferentes partes de la entrevista en estas categorías. En la 
primera se consideraron las respuestas a las preguntas de frases incompletas y de las fotografías de actividades. En la segunda, las preguntas de si recuerda las actividades de las fotografías, de las fotografías de las obras de teatro y de los videos, y en qué consisten las diferentes actividades. En el caso de los recuerdos, se agregó además una subcategoría según qué tanto apoyo necesitaba la persona para recordar: Recuerda sin apoyos, recuerda con apoyos de manera precisa, recuerda con apoyos de manera imprecisa y no recuerda.

A continuación, se presenta en la tabla 4 la clasificación de las preguntas realizadas según las categorías pre-establecidas. Los comentarios realizados por las personas participantes se categorizaron también en estas cuatro categorías según su definición.

Tabla 4

Definición de categorías de análisis y distribución de las preguntas según las categorías de análisis de la entrevista aplicada a participantes del Grupo de Teatro Rompecabezas entre noviembre de 2018 y febrero de 2019

\begin{tabular}{|c|c|c|}
\hline $\begin{array}{l}\text { Categorías } \\
\text { generales }\end{array}$ & Deffinición & Preguntas \\
\hline Satisfacción & $\begin{array}{l}\text { La persona determina si se } \\
\text { siente satisfecho o no con la } \\
\text { realización de una } \\
\text { actividad, manifestando } \\
\text { gusto o disgusto por ella. }\end{array}$ & $\begin{array}{l}\text { Frases incompletas } 1 \text { y } 2 \\
\text { Set de fotografías } 1,2 \text { y } 3 \text { : me gusta o no } \\
\text { me gusta. }\end{array}$ \\
\hline Apreciación & $\begin{array}{l}\text { La persona utiliza adjetivos } \\
\text { para caracterizar una } \\
\text { actividad }\end{array}$ & $\begin{array}{l}\text { Frases incompletas } 3,4,7 \text { y } 8 \\
\text { Set de fotografías } 1,2 \text { y } 3 \text { : actividad } \\
\text { favorita y actividad que menos le gusta. }\end{array}$ \\
\hline Recuerdos & $\begin{array}{l}\text { La persona describe lo que } \\
\text { recuerda sobre actividades } \\
\text { realizadas }\end{array}$ & $\begin{array}{l}\text { Set de fotografías } 1,2 \text { y } 3 \text { : recuerdas qué } \\
\text { hacíamos allí. } \\
\text { Set de fotografías } 4 \text { : recuerdos de las } \\
\text { obras de teatro y personajes. } \\
\text { Video de clase: recuerdo de actividades } \\
\text { realizadas. } \\
\text { Video de la obra: recuerdo de la obra } \\
\text { realizada }\end{array}$ \\
\hline Aprendizajes & $\begin{array}{l}\text { La persona determina } \\
\text { aquellos aprendizajes o } \\
\text { beneficios que obtiene en } \\
\text { las diferentes actividades } \\
\text { y/o define en qué consiste } \\
\text { un ejercicio propio del teatro }\end{array}$ & $\begin{array}{l}\text { Frases incompletas } 5 \text { y } 6 \\
\text { Set de fotografías } 1,2 \text { y } 3 \text { : nombre de los } \\
\text { ejercicios o actividades en teatro } \\
\text { presentadas en las fotografías. } \\
\text { Video de clase: descripción de en qué } \\
\text { consiste la actividad realizada en teatro. }\end{array}$ \\
\hline
\end{tabular}

Fuente: Elaboración propia, basado en las categorías de análisis aplicadas a las entrevistas realizadas entre noviembre 2018- febrero 2019.

Para presentar en igualdad de condiciones las respuestas de todas las personas participantes, se calculó la frecuencia con que cada participante dio un tipo de respuesta, determinando cuántos eligieron la misma categoría de respuesta para cada pregunta. Para 
calcular estos datos, las respuestas directas fueron procesadas a través de un paquete libre de estadística para ciencias sociales (PSPP), a través del cual se calcularon frecuencias de respuesta (directas y porcentajes calculados sobre las respuestas de 18 personas) y se procesaron las variables psicosociales para caracterizar al grupo de participantes. Estos datos se utilizan para describir los resultados obtenidos y no se realiza ningún tipo de inferencias estadísticas a partir de estos. Se utilizan de respaldo observaciones realizadas durante las entrevistas y la revisión del video de las mismas.

\section{Resultados}

Los resultados obtenidos de la entrevista se dividieron en dos categorías. Los primeros, relativos a la apreciación y satisfacción de las personas participantes sobre las actividades que se realizan dentro del proyecto en general y dentro de las clases de teatro en particular. Además, se indagaron beneficios que creen que obtienen de participar del proyecto y emociones asociadas a diferentes momentos. Los segundos resultados hacen referencia a los recuerdos y aprendizajes que tienen las personas participantes de las diversas actividades: en qué consisten, qué hacen en ellas.

A continuación, se presentan las frecuencias de respuestas asociadas a las preguntas de cada uno de estos grupos. Posteriormente, se presentará una discusión en la cual se analizan los resultados encontrados con base en la clasificación de grupos detallada anteriormente en la sección de participantes: respuestas del grupo completo; según año, actividades y obras en las que participaron; o respuestas únicamente de las personas con TEA por tratarse de temas vinculados con lo que se espera o no de esta población.

Con respecto a la apreciación de las personas participantes sobre las actividades, se obtuvieron los siguientes resultados. Al preguntarles por tres actividades diferentes (teatro, cocina y deporte), 55,56\% (10) señalaron que, de las tres, la que más le gusta es el teatro. Por otra parte, $23,53 \%$ (4) señalaron que el teatro es el que menos le gusta de las tres. Cabe señalar que, de ese porcentaje, conformado por cuatro participantes, gracias a esta entrevista se habló con sus familias y dos se retiraron del proyecto al finalizar su primer año porque realmente no les gustaba la actividad que realizaban. Los otros disfrutaban parte del proceso, aunque el teatro no les gustara en particular.

En cuanto a las actividades que se realizan en las sesiones de teatro (calentamiento, baile y montaje de escenas), sobre la actividad escogida como que les gusta realizar no hubo una tendencia marcada hacia alguna de las opciones, dividiéndose las respuestas en 
calentamiento con $27,78 \%$ (5), baile con 33,33\% (6) y practicar escenas con 38,89\% (7). Al preguntarles por esas mismas actividades cuál les gusta menos, la que obtuvo más puntaje fue calentamiento con $58,82 \%$ (10).

Al preguntarles individualmente por las actividades de las sesiones, mostrándoles fotos de cada una, $77,8 \%$ (14) señaló que les gustaba el calentamiento contra 16,7\% (3) que dijeron que no. Un participante no pudo escoger ninguna. A $88,89 \%(16)$ les gusta el baile contra $11,11 \%$ (2) a quienes no. En cuanto a practicar escenas, 83,33\% (15) dicen que les gusta contra 16,67\% (3) que no. Al escoger entre las opciones brindadas sobre cuál es su actividad favorita en clase, $55,56 \%$ (10) eligieron el baile, $27,78 \%$ (5) el calentamiento y $16,67 \%$ (3) practicar las escenas. Al escoger la que menos les gusta, 44,4\% (8) escogieron el calentamiento, contra $22,2 \%$ (4) respectivamente para baile y practicar escenas. Dos participantes no pudieron escoger una actividad como la que menos le gusta, encogiéndose de hombros y negándose a elegir. Estos resultados se resumen en la tabla 5.

\section{Tabla 5}

Frecuencia y porcentajes de respuestas a las preguntas relacionadas con el tipo de actividades realizadas en clase en la entrevista aplicada a participantes del Grupo de Teatro Rompecabezas entre noviembre de 2018 y febrero de 2019

\begin{tabular}{|c|c|c|c|c|}
\hline $\begin{array}{l}\text { Actividades } \\
\text { en clase }\end{array}$ & $\begin{array}{l}\text { De las tres, } \\
\text { ¿cuál le gusta } \\
\text { hacer? }\end{array}$ & $\begin{array}{l}\text { De las tres, } \\
\text { ¿cuál no le } \\
\text { gusta hacer? }\end{array}$ & $\begin{array}{l}\text { Entre las tres, ¿cuál } \\
\text { es tu favorita? }\end{array}$ & $\begin{array}{l}\text { Entre las tres, } \\
\text { ¿cuál te gusta } \\
\text { menos? }\end{array}$ \\
\hline Calentamiento & $5(27,78 \%)$ & $10(55,6 \%)$ & $5(27,78 \%)$ & $8(44,4 \%)$ \\
\hline $\begin{array}{l}\text { Practicar } \\
\text { escenas }\end{array}$ & $6(33,33 \%)$ & $4(22,2 \%)$ & $3(16,67 \%)$ & $4(22,2 \%)$ \\
\hline Bailar & 7 (38,89\%) & $3(16,7 \%)$ & $10(55,56 \%)$ & $4(22,2 \%)$ \\
\hline No responden & 0 & $1(5,6 \%)$ & 0 & $2(11,1 \%)$ \\
\hline Total & $18(100 \%)$ & $18(100 \%)$ & $18(100 \%)$ & $18(100 \%)$ \\
\hline
\end{tabular}

Fuente: Elaboración propia, información de frecuencias calculadas de las repuestas a las entrevistas a participantes del proyecto realizadas entre noviembre 2018- febrero 2019.

Como se puede apreciar en la tabla 5, realmente las tres actividades les gustan a las personas participantes, aunque hay una tendencia marcada entre las tres a que el calentamiento sea la que menos les gusta y el baile tenga más adeptos, incluso por encima de practicar escenas.

Se les preguntó para qué creían que les ayudaba el teatro. De las opciones trabajar con amigos, ser valiente y divertirme, 55,56\% (10) señaló que para lo que más le ayuda es para trabajar con amigos. Divertirme fue señalado por $27,78 \%$ (5) y ser valiente $16,67 \%$ (3). Sobre 
cómo se sienten en el teatro, 66,67\% (12) respondieron que se sienten felices, $27,78 \%$ (5) nerviosos y 5,56\% (1) triste. Al intentar indagar por qué triste, la persona que reportó esta emoción se rio y no quiso responder más, negando con la cabeza. Luego se evaluó con su padre y su madre, y no ha manifestado malestar con el proceso en el teatro, lo que tampoco se identificó por medio de observación ni en preguntas después de la evaluación, por lo que no se pudo clarificar esta respuesta.

También se les preguntó que podían hacer en la presentación. Las opciones fueron triunfar con mis compañeros, hacer reír a la gente y presentarse ante otras personas. La opción escogida con más frecuencia fue triunfar con mis compañeros con un 44,44\% (8), presentarme ante otras personas en segundo lugar con 33,33\% (6) y, en tercer lugar, hacer reír a la gente con $22,22 \%$ (4). Sobre cómo se sienten en la presentación, $61,11 \%$ (11) señaló que se sienten felices y $38,89 \%(7)$ se sienten nerviosos.

Con respecto al gusto por las diferentes actividades que se realizan en el proyecto, $94,44 \%$ (17) señaló que les gusta la clase. De estos, el 100\% (13) de las personas con TEA dijeron que les gusta la clase. Tan solo una persona, con otra situación de discapacidad, dijo que no le gustaba y se retiró tras un año de participación. Con respecto al gusto por hablar con otros en los espacios de receso o antes de clase, 94,44\% (17) señalaron que les gusta, solo uno de los participantes con TEA dijo no gustarle. Al 100\% (18) les gustan las actividades sociales como las celebraciones de cumpleaños o de fechas especiales, lo que manifestaron con sonrisas y asentimientos pronunciados. De esta forma, se manifiesta un gusto generalizado por las diversas actividades relacionadas con el proyecto, sean o no las clases de teatro en sí.

Al comparar estas tres actividades, las celebraciones sociales fueron la actividad favorita del grupo con 55,56\% (10) frente a 22,22\% (4) que prefieren hablar con otros en el receso o el ensayo. Entre las personas con TEA, 38,77\% (5) escogieron las celebraciones sociales, $30,77 \%$ (4) hablar con otros entre clases y $30,77 \%$ (4) el ensayo o clase. Este tipo de respuestas se destacan dado que señalan una diferencia con la idea instaurada de que las personas con TEA no les gusta la interacción social. Al preguntarles de las tres actividades cuál les gusta menos, entre la totalidad de participantes, 44,4\% (8) escogieron la clase con respecto a $33,3 \%$ (6) que escogieron hablar con otros entre clase. Tan solo para $16,7 \%$ (3) la actividad que menos les gusta son las celebraciones sociales. Un participante no pudo escoger cuál le gustaba menos. 
En cuanto a las actividades fuera del teatro, 83,33\% (15) dijeron que les gustan las actividades de prensa y divulgación como hablar con periodistas. Parece manifestarse así un gusto en la mayoría del grupo de proyectar su voz a otras personas a través de medios de comunicación masiva.

Las dos otras actividades que se evaluaron fue solo con los seis participantes, todos con TEA, que habían participado de las mismas al haber estado más tiempo en el proyecto. El $100 \%$ (6) señalaron que les gusta participar de las grabaciones para audiovisuales y de las sesiones de tecnologías abiertas en el laboratorio de fabricación Kä Träre de la UNED.

Además de los gustos por las actividades realizadas, se indagaron los recuerdos y aprendizajes de las personas participantes para tener certeza de que estaban claros sobre qué actividades se les estaba preguntando. Para ello, se utilizaron tres medios diferentes: uno, preguntando qué ocurre en cada fotografía que se presentó para acompañar las opciones de respuesta de las preguntas anteriores; dos, con fotografías de las obras de teatro en las que han participado y tres, presentando videos de ejercicios o de obras de teatro en las que aparecía la persona y preguntándole que sucedía.

En cuanto a identificar fotografías, los resultados fueron los siguientes. En relación con las fotografías de las clases, 44,44\% (8) identifica las clases de teatro directamente. Otro $44,44 \%$ (8) la identifica con apoyos por parte de la entrevistadora y solo dos personas no la identificaron. En lo relacionado con hablar con otros en los recesos, todos lo identificaron, $61,11 \%$ (11) sin apoyos y 38,89\% (7) con apoyos. Al respecto de las actividades sociales, $88,89 \%$ (16) las identificaron sin ayuda, una persona con apoyos y otra no las identificó del todo.

Con respecto a identificar el calentamiento, $66,67 \%$ (12) lo identificaron, $22,22 \%$ (4) lo hicieron con apoyos y $11,1 \%$ (2) no lo identificaron. Acerca de la fotografía de baile, $88,89 \%$ (16) la identificó sin problemas y 11,11\% (2) con apoyo. Al identificar la fotografía de practicar escenas, $70,59 \%$ (12) lo hicieron sin ayudas, y 29,41\% (5) con apoyo. Uno no la identificó.

Por su parte, en lo referente a prensa y divulgación, 66,67\% (12) reconoció la actividad y $27,78 \%$ (5) lo hizo con apoyos. Una persona no la identificó. En cuanto a las grabaciones, de los seis participantes que han formado parte de sesiones de grabar una obra de teatro, $66,67 \%$ (4) reconocieron la actividad sin ayuda, 16,67\% (1) respectivamente con apoyo o no la reconocieron. Las actividades de tecnología en el Fab Lab fueron reconocidas por el 100\% (6) de quienes participaron de ellas. 
Cuando una persona no recordaba del todo se le comentaba cuál actividad era para que pudiera indicar si le gustaba o no. Sin embargo, esto fue poco frecuente, dado que, como muestran las frecuencias, la mayoría reconoció siempre la foto o con algunas indicaciones supo de qué momento se trataba. Aquellos que no recordaron del todo son personas que, en otros momentos, ya sea en interacciones cotidianas o en actividades del teatro como la memorización de textos y marcaciones, suelen presentar dificultades de memoria.

En cuanto a recordar las obras de teatro, se le preguntó a cada participante lo que recordaba de la trama de la obra y de su papel en ella. En el caso de las seis personas que habían participado en las primeras cuatro obras de teatro, se les preguntó por cada una. Por su parte, al grupo de 12 personas que participó en la última, se les preguntó solamente sobre esta. Los resultados para recordar la trama de la obra se detallan en la tabla 6.

\section{Tabla 6}

Distribución de participantes según su facilidad para recordar la trama de las obras de teatro en las que participaron en la entrevista aplicada a participantes del Grupo de Teatro Rompecabezas entre noviembre de 2018 y febrero de 2019

\begin{tabular}{|c|c|c|c|c|c|}
\hline Trama de la obra & $\begin{array}{l}\text { Recordaron } \\
\text { sin ayuda }\end{array}$ & $\begin{array}{l}\text { Recuerda } \\
\text { con apoyos } \\
\text { de manera } \\
\text { precisa }\end{array}$ & $\begin{array}{l}\text { Recordaron } \\
\text { con apoyos } \\
\text { de manera } \\
\text { imprecisa }\end{array}$ & No recordaron & Total \\
\hline $\begin{array}{l}\text { Querido Santa } \\
(2016)\end{array}$ & $2(33,33 \%)$ & $0 \%$ & $2(33,33 \%)$ & $2(33,33 \%)$ & $6(100 \%)$ \\
\hline $\begin{array}{l}\text { El árbol de la vida } \\
\text { (2017) }\end{array}$ & $1(16,67 \%)$ & $0 \%$ & $3(50 \%)$ & $2(33,33 \%)$ & $6(100 \%)$ \\
\hline $\begin{array}{l}\text { Una navidad para } \\
\text { recordar (2017) }\end{array}$ & $0 \%$ & $2(33,33 \%)$ & $3(50 \%)$ & $1(16,67 \%)$ & $6(100 \%)$ \\
\hline Así soy yo (2018) & $1(16,67 \%)$ & $1(16,67 \%)$ & $2(33,33 \%)$ & $2(33,33 \%)$ & $6(100 \%)$ \\
\hline $\begin{array}{l}\text { El día que las brujas } \\
\text { intentaron robarse } \\
\text { la Navidad (2018) }\end{array}$ & $6(50 \%)$ & $1(8,33 \%)$ & $5(41,67 \%)$ & $0 \%$ & $12(100 \%)$ \\
\hline
\end{tabular}

Fuente: Elaboración propia, información de frecuencias calculadas de las repuestas a las entrevistas a participantes del proyecto realizadas entre noviembre 2018- febrero 2019.

Como se puede ver, en el caso del grupo que participó en las cuatro obras de teatro, son pocos quienes recuerdan sin ayuda la trama, por lo general necesitan de apoyos para ello, en especial aquellas que hicieron hace más tiempo. La obra que ninguno recuerda de manera precisa es aquella que tuvo menos sesiones de montaje y una única presentación, en contraste con las otras que habían ejecutado al menos dos veces.

En cuanto a la quinta obra de teatro, presentada solo por el grupo más nuevo en el que hay personas con diversas situaciones, todos recordaron en alguna medida la trama. Debe considerarse que al realizar la entrevista era la única obra de teatro que habían presentado y 
apenas habían pasado de uno a dos meses, contrario a los otros que habían presentado cuatro obras de teatro a lo largo de cuatro años, la última de ellas más de dos meses antes.

En cuanto a la memoria relacionada con los personajes que interpretaba la persona en las obras de teatro, se presentan los resultados en la tabla 7.

Tabla 7

Porcentaje de frecuencia de recuerdo sobre el personaje que interpretaban en cada una de las obras de teatro en la entrevista aplicada a participantes del Grupo de Teatro Rompecabezas entre noviembre de 2018 y febrero de 2019

\begin{tabular}{|c|c|c|c|c|c|}
\hline $\begin{array}{l}\text { Personaje de la } \\
\text { obra }\end{array}$ & $\begin{array}{l}\text { Recordaron } \\
\text { sin ayuda }\end{array}$ & $\begin{array}{l}\text { Recuerda } \\
\text { con apoyos } \\
\text { de manera } \\
\text { precisa }\end{array}$ & $\begin{array}{l}\text { Recordaron } \\
\text { con apoyos } \\
\text { de manera } \\
\text { imprecisa }\end{array}$ & No recordaron & Total* \\
\hline $\begin{array}{l}\text { Querido Santa } \\
(2016)\end{array}$ & $2(33,33 \%)$ & $0 \%$ & $2(33,33 \%)$ & $2(33,33 \%)$ & $6(100 \%)$ \\
\hline $\begin{array}{l}\text { El árbol de la vida } \\
(2017)\end{array}$ & $1(16,67 \%)$ & $0 \%$ & $4(66,67 \%)$ & $1(16,67 \%)$ & $6(100 \%)$ \\
\hline $\begin{array}{l}\text { Una navidad para } \\
\text { recordar (2017) }\end{array}$ & $2(33,33 \%)$ & $1(16,67 \%)$ & $3(50 \%)$ & $0 \%$ & $6(100 \%)$ \\
\hline Así soy yo (2018) & $1(16,67 \%)$ & $1(16,67 \%)$ & $4(66,67 \%)$ & $0 \%$ & $6(100 \%)$ \\
\hline $\begin{array}{l}\text { El día que las brujas } \\
\text { intentaron robarse } \\
\text { la Navidad (2018) }\end{array}$ & $8(66,67 \%)$ & $4(33,33 \%)$ & $0 \%$ & $0 \%$ & $12(100 \%)$ \\
\hline
\end{tabular}

*Total de personas entrevistadas que participaron en cada obra de teatro

Fuente: Elaboración propia, información de frecuencias calculadas de las repuestas a las entrevistas a participantes del proyecto realizadas entre noviembre 2018- febrero 2019.

Finalmente, a todas las personas participantes se les mostró un video de la clase, para corroborar lo visto en las fotografías de que recordaban las actividades, esta vez en un formato audiovisual. En todos los casos se usó un video en el que apareciera la persona entrevistada y todos correspondían a ejercicios durante el calentamiento. Al ver el video, 55,56\% (10) recordó lo que hacían, 38,89\% (7) lo recordó con apoyos y tan solo una persona, 5,56\% (1) no supo decir qué hacían en el momento del video.

Estos resultados permiten observar que las personas participantes recuerdan y reconocen los espacios principales de las actividades del taller de teatro: baile, calentamiento y ensayo. Pueden describir en qué consisten y opinar sobre ellas, aunque algunas personas necesiten apoyo para recordar momentos y actividades específicas. Esto no es extraño ni ajeno a lo encontrado en investigaciones anteriores donde se señaló encontrar dificultades de memoria con algunas personas con TEA al realizar entrevistas, aunque no fuera generalizado (Harrington et al., 2014). Cabe destacar que estos resultados varían entre el grupo 1 compuesto por seis personas con diagnóstico de TEA con respecto al grupo 2 compuesto por 12 personas con diferentes situaciones de discapacidad. 
Esto fue especialmente evidente al pedirles recordar las obras de teatro en las que habían participado y el papel de su personaje. Se planteó la hipótesis de que el ensayo fragmentado de la obra completa al tener que dividirla en escenas y en las diferentes participaciones, así como no siempre se ensaya en el orden que van las escenas, no ayudan a que la persona pueda retener la trama completa. Esta información extraída de las entrevistas se llevó a las sesiones, incorporando al trabajo semanal un espacio para recordar la trama y mensaje de la obra cada cierto número de ensayos. Esto constituye un claro ejemplo de cómo escuchar la voz de las personas participantes facilita la mejora del trabajo en el proyecto.

En lo que se refiere a la apreciación por las actividades realizadas, los resultados evidencian un gusto generalizado tanto por el teatro en sí como por las acciones que se desarrollan en él. Llama la atención como las actividades grupales y de interacción fuera de la clase representan las que la mayoría seleccionaron como las que más disfrutaban: el baile, hablar con otros en los espacios de receso o antes de clase, y las actividades sociales como las celebraciones de cumpleaños o de fechas especiales.

Aunado a lo anterior, la mayoría de las opiniones de los y las participantes en relación con los beneficios que el teatro les aportaba, iban enfocadas al trabajo con amigos [55,56\% (10)] y la diversión [27,78\% (5)]. Mientras que lo que las presentaciones ante el público les permitía hacer, las opciones más escogidas por las personas participantes fueron las de triunfar con sus compañeros [44,44\% (8)], y presentarse ante otras personas [33,33\% (6)].

Contrario a la creencia popular y los mitos que giran alrededor de las personas con TEA, si bien las dificultades en la comunicación y la interacción social son propias de la condición (Alcantud, Rico, y Lozano, 2012), las opiniones de las personas participantes del proyecto, que se pueden apreciar al separar las respuestas de las personas con TEA de las demás, evidencian que las mismas no anulan o suprimen el deseo, interés y agrado por participar de actividades sociales y establecer relaciones afectivas con otros cuando se les brinda la oportunidad.

Otra de las impresiones erróneas de la realidad de estas personas, es la de que no son capaces de expresar sus sentimientos, comunicarse o relacionarse con los demás (Hijosa, 2017). Sin embargo, las respuestas de los y las participantes reflejaron la existencia de un deseo y un gusto por expresarse y compartir con otros sobre aspectos y experiencias de sus vidas. Más de la mitad dijeron haberles gustado participar en actividades de prensa y divulgación, así como grabación de audiovisuales, lo cual ha involucrado el hablar con periodistas y personas desconocidas, en ambientes familiares y ajenos a su contexto, estar 
frente a cámaras, luces y micrófonos; todas experiencias que, por los criterios diagnósticos del TEA relacionados con las rutinas y el procesamiento sensorial, podría asumirse que no pueden participar o no disfrutan hacerlo.

Con respecto a la expresión de sus gustos e intereses en las actividades realizadas, fue clara la seguridad con la que cada participante definió las cosas que le gustaban y para elegir qué preferían entre varias opciones. Sin embargo, no hubo la misma seguridad y contundencia para responder a las preguntas sobre qué no les gusta. Algunos autores han reportado para la entrevista con jóvenes con TEA una dificultad de parte de algunos de ellos para relacionar sus propias opiniones con aspectos subjetivos (Brewster \& Coleyshaw, 2011). Sin embargo, esta dificultad no puede convertirse en un límite que impida buscar formas de escuchar la voz de las personas participantes, aunque no puede dejar de considerarse como un reto.

La dificultad de las personas participantes en este estudio para dar respuesta a qué no les gusta podría evaluarse desde otras perspectivas también. Por ejemplo, aunque existen instrumentos para medir la deseabilidad social que pueda mediar las respuestas, es una dificultad en general para la investigación, dado que puede comprometer la validez y confiabilidad de un estudio (Ranjan y George, 2014) pero no se encuentra información específica de cómo evaluarla en instrumentos diseñados para personas en situación de discapacidad, ni siquiera en sistematizaciones propias de cómo realizar investigación con la población (Fayette y Bond, 2018).

Otro punto para valorar es la posibilidad de que no tengan la costumbre de que se les valide que una intervención pueda no gustarles, dado que cuando alguna de las actividades no les gustaba, no respondían de inmediato, sino que mostraban duda e inquietud, y al reasegurarles que no había problema si una actividad no les gustaba y es normal que no a todas las personas les guste lo mismo, reportaban no ser de su agrado.

La experiencia descrita ha sido un primer intento de abrir espacio a la voz de los y las participantes. En este caso se ha centrado únicamente en conocer su satisfacción y apreciación con las actividades realizadas en el proyecto de teatro, así como explorar los recuerdos y aprendizajes adquiridos. No obstante, un mayor involucramiento y una participación más allá de la opinión, es un reto y tema pendiente.

Como se expone en los antecedentes, las investigaciones sobre el tema de teatro y TEA se han desarrollado desde la posición de personas expertas hacia la población en situación de discapacidad, y solo en algunos casos aparecen las personas con TEA como informantes directos. 
Existe por lo tanto la necesidad de una mayor exploración de los métodos y estrategias a utilizar en las investigaciones que involucran personas en situación de discapacidad, de forma que estén comprometidas con escuchar de manera auténtica, darles la voz y autoridad para profundizar en los procesos de construcción, análisis e interpretación de los datos (Susinos y Parrilla, 2013). Incluso se requiere una participación más activa sobre la toma de decisiones al respecto de las temáticas a explorar, que den paso a otras líneas de investigación y reflejen realmente sus preocupaciones, intereses y demandas sobre su realidad.

De igual forma, al trabajar desde el arte, como es el teatro, hay que eliminar las concepciones de este como algo abstracto y ajeno al entendimiento o participación de las personas en situaciones de discapacidad como el TEA. Este trabajo y el disfrute que manifiestan las personas participantes muestran que son capaces de hacer teatro y disfrutarlo desde sus propias capacidades, habilidades e intereses, y resalta la importancia de recordar que el acceso al arte es un derecho de las personas, independientemente de sus situaciones o condiciones. No se les debe negar la oportunidad de explorarlo desde su propia experiencia por un diagnóstico, de igual forma que no se les debe imponer cómo hacer arte, sino facilitarles las posibilidades para hacerlo desde sí mismos.

\section{Conclusiones}

Ofrecer espacios para escuchar a las personas participantes de un proyecto es esencial para valorar, desde su punto de vista, los aciertos, las oportunidades de mejora y en qué dirección tomar decisiones. Las entrevistas estructuradas con apoyos permiten, dar voz y expresión, independientemente de su competencia verbal, a las personas participantes. A través de la metodología utilizada se pusieron en manifiesto los aspectos que se proponía evaluar en esta experiencia.

Lo anterior, aporta un valor añadido al proceso de investigación y evidencia lo propuesto por el modelo social de la discapacidad, de que lo que limita e impide que las personas en situación de discapacidad se incluyan en estos procesos y tengan la oportunidad de participar, comunicar y aportar, reside en las barreras que el contexto impone y la falta de apoyos para garantizar el goce o ejercicio de este derecho, en igualdad de condiciones que los demás (ONU, 2006 y Victoria, 2013).

En primer lugar, con respecto al objetivo de indagar la satisfacción de participar de este proceso de teatro, se encontró que la mayoría expresan gusto por el teatro, por las sesiones de trabajo y las actividades realizadas, así como se hizo explícito el deseo de la mayoría de 
continuar. Este gusto de la mayoría coincide con los hallazgos de otros autores con sus respectivos grupos de teatro (Loyd, 2013; Mehling et al, 2017) En cuanto a su apreciación sobre las actividades en las que participaron las experiencias más gustadas fueron los momentos de compartir con otros: actividades sociales, los recesos y las clases. Esto coincide con la forma en que las personas participantes del estudio de Loyd (2013) lograron explicar qué actividades les gustaban más y cuáles menos, e incluso asociar la emoción de felicidad a las actividades.

Con respecto al objetivo de explorar sus recuerdos del proceso, fue posible determinar que la mayoría puede recordar las obras de teatro en las que han participado de manera básica, así como sus personajes y el tema general de la obra. Lo mismo sucede con los diferentes ejercicios y actividades realizados en los talleres. En el caso del estudio de Loyd (2013) todos podían recordar estos aspectos. Algunas personas participantes tuvieron mayores dificultades para recordar, aunque fueron aquellas que en otros momentos y actividades también tienen esta limitación.

Con respecto a los aprendizajes alcanzados, más de la mitad del grupo hizo énfasis al elegir entre varias opciones en que el teatro le ha ayudado a trabajar con otras personas, aún cuando las otras opciones eran más individuales y enfocadas al éxito personal. De igual forma, casi la mitad del grupo destacó que al actuar puede triunfar con sus compañeros, en contraste con otras opciones de logros personales. Estas respuestas parecen apuntar al sentido de pertenencia al grupo que fue posible apreciar a lo largo de las entrevistas, así como al desarrollo de las habilidades sociales que busca el proceso. En otros estudios se evidenció que las personas participantes tenían claridad de la importancia del trabajo con otros para realizar teatro (Loyd, 2013), la mejora que este había implicado en sus relaciones (Kim y Boyns, 2015) y los beneficios que esta actividad trae a su vida (Blanco et al, 2016). También fue posible evidenciar que todas las personas participantes manejan conceptos y actividades propias del trabajo del teatro, de manera que pueden referirse a ellas e incluso explicarlas.

Con respecto al objetivo de visibilizar una metodología aplicada para recuperar los puntos de vista de todas las personas participantes, al utilizar múltiples maneras de presentar la información y permitir la expresión de las respuestas según las posibilidades de cada quien, demostró ser accesible a todas las formas de comunicación de los y las participantes, quienes incluían personas con lenguaje verbal oral así como aquellos que requerían apoyos dada su necesidad de formas aumentativas de la comunicación para poder expresarse, incluso uno cuya lengua materna era el inglés. Para lograr esto se utilizó la propuesta de Loyd (2013) con 
resultados similares en cuanto a que todas las personas participantes pudieron participar de todas las partes de la entrevista. La totalidad de las personas participantes lograron expresarse, valorar su proceso, manifestar sus gustos, preferencias y demostrar sus recuerdos del proceso dentro del Grupo de Teatro Inclusivo Rompecabezas. Se destaca por lo tanto esta experiencia, y las recopiladas como antecedentes de este trabajo (Preece y Jordan, 2009; Lewis, 2009; Danker, Strnadová y Cumming, 2017; Harrington, Foster, Rodger y Ashburner, 2013; Fletcher-Watson et al., 2018; Blanco, Regueiro y González, 2016; Mehling et al., 2017; Trowsdale y Hayhow, 2013; Kim y Boyns, 2015), que han permitido, con la planificación y selección del método y formato adecuado, que la voz de las personas en alguna situación de discapacidad pueda ser escuchada y valorada en procesos de construcción del conocimiento.

Como limitaciones en el estudio, debe tomarse en cuenta que no se contó con un instrumento adecuado para la medición de la deseabilidad social y que en el caso de las personas que el español no es su lengua materna, si bien se hizo la entrevista en inglés, no se contó con el apoyo de intérprete profesional. También debe considerarse que las personas entrevistadoras y entrevistadas tenían mínimo un año de trabajo conjunto en el Grupo de Teatro, lo que deben tomar en consideración otros investigadores al replicar la experiencia, en caso de que no exista el mismo tipo de vínculo entre ambas partes.

A partir de los resultados obtenidos por esta experiencia, fue posible, además, tomar en consideración lo aportado por las personas participantes para realizar modificaciones en la dinámica de trabajo y apoyar otras aristas de la investigación. Como se identificó que algunos tenían dificultades con recordar la trama de la obra, se inició la práctica de recordar durante el montaje la trama en varias ocasiones, para evitar que, al trabajarla en forma seccionada, cada persona ensayando sus escenas, se perdiera la esencia del mensaje que se estaba trabajando. Además, se identificó a personas que no les gustaban las actividades. Fueron escuchados por el equipo investigador y, posteriormente, por sus familias. A partir de esto, se pudo valorar en conjunto su continuidad y finalmente decidieron salir del proceso a buscar otras opciones, en especial porque no les gustaba la experiencia de teatro. Finalmente, las apreciaciones de las personas participantes en relación con su agrado, satisfacción, recuerdos y aprendizajes de la experiencia en el Grupo Rompecabezas, refuerza la propuesta del uso del teatro como una estrategia de mediación pedagógica (Sandoval-Poveda, González-Rojas y Madriz-Bermúdez, 2020).

Si bien es un proceso que puede resultar retador, la invitación de las autoras a otros grupos investigadores de diversas áreas es la de replicar este tipo de experiencias y buscar 
opciones para escuchar la valoración propiamente de las personas beneficiarias directas, y no solamente de sus familiares, profesionales o las observaciones de terceros. Esta experiencia pone en evidencia tanto que la metodología aplicada en el grupo Rompecabezas está siendo valorada positivamente por sus participantes como la oportunidad que este tipo de entrevistas brinda para los procesos de investigación, ya sea desde educación o de otras áreas. Asimismo, es necesario aumentar, con los apoyos y ajustes razonables que se requieran, el desarrollo de investigaciones más inclusivas, en las que las personas en alguna situación de discapacidad se vean realmente representadas, con roles más activos como diseñadores y responsables de proyectos centrados en el aporte de soluciones y en la mejora de su calidad de vida.

\section{Agradecimientos}

A las personas participantes y sus familias, por su confianza y consentimiento de participación en este proyecto; y porque cada una con sus aportes, han sido piezas clave de este rompecabezas.

\section{Referencias}

Alcantud, Francisco., Rico, Darío., y Lozano, Castro. (2012). Trastornos del Espectro Autista. Guía para padres y profesionales. València: Centre Universitari de Diagnòstic i Atenció Primerenca, Universitat de València.

Asociación Americana de Psiquiatría. (2013). Guía de consulta de los criterios diagnósticos del $D S M-5^{T M}$. Arlington, VA: Asociación Americana de Psiquiatría.

Beresford, Bryony., Tozer, Rosemary., Parvaneh, Rabiee., y Sloper, Patricia. (2004). Developing an approach to involving children with autistic spectrum disorders in a social care research project. British Journal of Learning Disabilities, 32(4), 180-85. doi: https://doi.org/10.1111/j.1468-3156.2004.00318.x

Beadle-Brown, Julie., Wilkinson, David., Richardson, Lisa., Shaughnessy, Nicola., Trimingham, Melissa., Leigh, Jennifer., Whelton, Beckie., y Himmerich, Julian. (2018). Imagining Autism: Feasibility of a drama-based intervention on the social, communicative and imaginative behaviour of children with autism. Autism, 22(8), 915-927. doi: https://doi.org/10.1177/1362361317710797

Blanco, Alfredo., Regueiro, Jessica y González, Mercedes. (2016). El teatro como herramienta socializadora para personas con Asperger. Revista Española de Orientación y Psicopedagogía, 27(2), 116-125. Recuperado de http://www.redalyc.org/articulo.oa?id=338246883008 
Brewster, Stephanie. y Coleyshaw, Liz. (2011). Participation or exclusion? Perspectives of pupils with autistic spectrum disorders on their participation in leisure activities. British Journal of Learning Disabilities, 39(4), 284-291. doi: 10.1111/j.1468-3156.2010.00665.x

Bustamante, Jazmín., Miranda, Javiera. y Sánchez, Susan. (2020). Pedagogía teatral en el desarrollo de habilidades sociales de los estudiantes de primero y segundo año básico con y sin necesidades educativas especiales de una escuela con dependencia Municipal de alta vulnerabilidad (Tesis de grado en educación diferencial). Universidad de Concepción, Los Ángeles, Chile. Recuperado de http://152.74.17.92/bitstream/11594/680/1/Bustamante\%20Miranda\%20Sanchez.pdf

Calafat-Selma, María., Sanz-Cervera, Pilar. y Tárraga-Mínguez, Raúl. (2016). El teatro como herramienta de intervención en alumnos con trastorno del espectro autista y discapacidad intelectual. Revista Nacional e Internacional de Educación Inclusiva, 9(3), 95-108.

Cascio, M. Ariel., Weiss, Jonathan. y Racine, Éric. (2021). Making Autism Research Inclusive by Attending to Intersectionality: a Review of the Research Ethics Literature. Rev J Autism Dev Disord 8, 22-36. https://doi.org/10.1007/s40489-020-00204-z

Cook, Angelle. (2020). Using an inclusive therapeutic theatre production to teach self-advocacy skills in young people with disabilities. The Arts in Psychotherapy, 71,. doi:10.1016/j.aip.2020.101715

Corbett, Blythe A., Blain, Scott D., Ioannou, Sara., and Balser, Maddie. (2017). Changes in anxiety following a randomized control trial of a theatre-based intervention for youth with autism spectrum disorder. Autism, 21(3), 333-343. doi:10.1177/1362361316643623

Corbett, Blythe A., Key, Alexandra P., Qualls, Lydia., Fecteau, Stephanie., Newsom, Cassandra., Coke, Catherine., y Yoder, Paul. (2016). Improvement in Social Competence Using a Randomized Trial of a Theatre Intervention for Children with Autism Spectrum Disorder. Journal of Autism and Developmental Disorders, 46(2), 658-672. doi:10.1007/s10803-015-2600-9

Corbett, Blythe A., Swain, Deanna M., Coke, Catherine., Simon, David., Newsom, Cassandra., Houchins-Juarez, Nea., Jenson, Ashley... y Song, Yanna. (2014). Improvement in social deficits in autism spectrum disorders using a theatre-based, peer-mediated intervention. Autism Research, 7(1), 4-16. doi:10.1002/aur.1341

Danker, Joanne., Strnadová, Iva. y Cumming, Therese M. (2017). Engaging Students with Autism Spectrum Disorder in Research Through Participant-Driven Photo-Elicitation Research Technique. Australasian Journal of Special Education, 41(1), 35-50. doi: 10.1017/jse.2016.7

Fayette, Rainart y Bond, Caroline (2018). A systematic literature review of qualitative research methods for eliciting the views of young people with ASD about their educational experiences. European Journal of Special Needs Education, 33(3), 349-365. doi:10.1080/08856257.2017.1314111

Fernández, María., Guerra, Patricia. y Begara, Olaya. (2013). El teatro como instrumento terapéutico en la rehabilitación psicosocial. Norte de salud mental, 11(42), 82-88. 
Fletcher-Watson, Sue., Adams, Jon., Brook, Kabie., Charman, Tony., Crane, Laura., Cusack, James., Leekam, Susan., Milton, Damian., Parr, Jeremy R., y Pellicano, Elizabeth. (2018). Making the future together: Shaping autism research through meaningful participation. Autism, 23(4), 943-53. doi: 10.1177/1362361318786721.

Fullana, Judit, María Pallisera, Montserrat Vilà, y Carolina Puyaltó. (2016). Las personas con discapacidad intelectual como investigadoras. Debates, retos y posibilidades de la investigación inclusiva. EMPIRIA. Revista de Metodología de Ciencias Sociales 0(33),111-38. doi:10.5944/empiria.33.2016.15866

García, María. (2014). Teatro y discapacidad intelectual (Trabajo fin de grado en Psicología, Facultad de Humanidades y Ciencias de la Educación). Universidad de Jaén, Jaén, España.

Recuperado

de http://tauja.ujaen.es/bitstream/10953.1/1075/4/TFG_Garc\%c3\%adaS\%c3\%a1nchez\%2c Mar\%c3\%adaJos\%c3\%a9.pdf

González, Natalia. (2017). El Teatro Pedagógico como modelo de intervención en las Necesidades Educativas Específicas para personas con Síndrome de Down (Tesis doctoral). Universidad de La Laguna, España. Recuperada de https://riull.ull.es/xmlui/handle/915/7627

González, María. (2018). El desarrollo de competencias mediante el Teatro Pedagógico: Un estudio de caso de una persona con síndrome de Down (Tesis de Máster en intervención psicopedagógica en contextos de educación formal y no formal). Recuperado de https://riull.ull.es/xmlui/handle/915/10854

Guli, Laura A., Semrud-Clikeman, Margaret., Lerner, Matthew D., y Britton, Noah. (2013). Social Competence Intervention Program (SCIP): A pilot study of a creative drama program for youth with social difficulties. Arts in Psychotherapy, 40(1), 37-44. doi:10.1016/j.aip.2012.09.002

Harrington, Caitlin., Foster, Michele., Rodger, Sylvia., y Ashburner, Jill. (2014). Engaging young people with autism spectrum disorder in research interviews. British Journal of Learning Disabilities, 42(2), 153-161. doi:10.1111/bld.12037

Heeks, Bill. (2015). Theatre and dramatherapy in health education and autism. Godd Autism Practice, 16(2), 21-25. Recuperado de https://www.ingentaconnect.com/contentone/bild/gap/2015/00000016/00000002/art0000 4;jsessionid=1 neczuiihn7g2.x-ic-live-02

Hens, Kristien., Robeyns, Ingrid., y Schaubroeck, Katrien. (2019). The ethics of autism. Philosophy Compass, 14(1), 1-16. doi:10.1111/phc3.12559

Hijosa, Laura. (2017). Guía informativa para familias de personas con trastorno del espectro del autismo. Madrid, España: Comunidad de Madrid, Consejería de Políticas Sociales y Familia, Dirección General de Atención a Personas con Discapacidad. Recuperado de http://www.madrid.org/bvirtual/BVCM014017.pdf

loannou, Sara., Key, Alexandra P., Muscatello, Rachael A., Klemencic, Mark., y Corbett, Blythe A. (2020). Peer Actors and Theater Techniques Play Pivotal Roles in Improving Social Play and Anxiety for Children With Autism. Frontiers in Psychology, 11, 1-9. doi:10.3389/fpsyg.2020.00908 
Kempe, Andy. y Tissot, Cathy (2012). The use of drama to teach social skills in a special school setting for students with autism. British Journal of Learning Support, 27(3),97-102. doi: 10.1111/j.1467-9604.2012.01526.x

Kim, Ah-Jeong., y Boyns, David. (2015). Joining The Spectrum: An Interdisciplinary Inquiry Into Theatre As An Intervention For Autism Diagnosed Teens. Recuperado de https://www.arts.gov/sites/default/files/Research-Art-Works-CSUNorthridge.pdf

Kim, Ah-Jeong., Stembridge, Sarah., Lawrence, Christopher., Torres, Vincent., Miodrag, Nancy., Lee, Joong-Won., Boyns, David. y Boyns, David (2015). Neurodiversity on the Stage: The Effects of Inclusive Theatre on Youth with. International Journal of Education and Social Science, 2(9), 27-39. Recuperado de http://www.ijessnet.com/wpcontent/uploads/2015/10/4.pdf

Lenakakis, Antonis. y Koltsida, María. (2017). Disabled and non-disabled actors working in partnership for a theatrical performance: a research on theatrical partnerships as enablers of social and behavioural skills for persons with disabilities. Research in Drama Education: The Journal of Applied Theatre and Performance, 22(2), 251269. doi:10.1080/13569783.2017.1286975

Lewis, Ann. (2009). Methodological issues in exploring the ideas of children with autism concerning self and spirituality. Journal of Religion, Disability and Health 13(1), 64-76. doi: $10.1080 / 15228960802581446$.

Loftis, Sonya. (2019). Autistic Culture, Shakespeare therapy and the Hunter Heartbeat Method. Shakespeare Survey, 72, 256-267. doi: $10.1017 / 9781108588072.020$

Loyd, Daisy. (2013) Gaining views from pupils with autism about their participation in drama classes. British Journal of Learning Disabilities, 43, 8-15. doi: 10.1111/bld.12078

Mehling, Margaret H., Tassé, Marc., y Root, Robin. (2017). Shakespeare and autism: an exploratory evaluation of the Hunter Hertbeat Method. Research and Practice in Intellectual and Developmental Disabilities, 4(2), 107-120. doi:10.1080/23297018.2016.1207202

Ministerio de Sanidad Servicios Sociales e Igualdad. (2019). Estrategia Española en Trastornos del Espectro del Autismo. España. Recuperado de https://www.mscbs.gob.es/ssi/discapacidad/informacion/estrategiaEspanolaAutismo.htm

Narváez, Estefanía., Parra, Claudia., y Sepúlveda, Pilar. (2020). Una intervención teatral para incrementar las habilidades sociales en estudiantes con necesidades educativas especiales múltiples en una escuela especial. Paideia, (54), 99-114. Recuperado de http://revistasacademicas.udec.cl/index.php/paideia/article/view/1689

Neulfeld, David. (2012). Integrated Drama Groups: Promoting Symbolic Play, Empathy, and Social Engagement With Peers in Children with Autism (Tesis doctoral). Recuperada de https://digitalassets.lib.berkeley.edu/etd/ucb/text/Neufeld_berkeley_0028E_12375.pdf 
Organización de las Naciones Unidas (ONU). (2006). Convención sobre los derechos de las personas con discapacidad. Recuperado de https://www.un.org/esa/socdev/enable/documents/tccconvs.pdf

Palacios, Agustina. (2008). El modelo social de la discapacidad: Orígenes, caracterización y plasmación en la Convención Internacional sobre los Derechos de las Personas con Discapacidad. Madrid: Grupo Editorial CINCA. Recuperado de https://www.cermi.es/sites/default/files/docs/colecciones/Elmodelosocialdediscapacidad. pdf

Pallisera, María., Fullana, Judit., Puyaltó, Carol., Vilà, Montserrat., y Díaz, Gemma. (2017). Apoyando la participación real de las personas con discapacidad intelectual: una experiencia de investigación inclusiva sobre vida independiente. Revista Española de Discapacidad, 5(1),7-24. doi: 10.5569/2340.

Pallisera, María., y Puyalto, Carolina. (2014). La voz de las personas con discapacidad intelectual en la investigación: hacia el desarrollo de investigaciones inclusivas. Revista de Educación Inclusiva, 7(2),84-97.

Pallisera, Maria., Puyaltó, Carolina., Fullana, Judit., Vilà, Montserrat., y Martin, Raquel. (2015). Una experiencia de investigación inclusiva. Personas con discapacidad intelectual como asesoras en una investigación sobre transición a la edad adulta. Revista Iberoamericana de Educación, 69(2),147-66. Recuperado de http://riberdis.cedd.net/bitstream/handle/11181/4819/Una_experiencia_de_investigacion _inclusiva.pdf?sequence $=1$

Preece, David. y Jordan, Rita. (2010). Obtaining the views of children and young people with autism spectrum disorders about their experience of daily life and social care support. British Journal of Learning Disabilities 38(1), 10-20. doi: 10.1111/j.14683156.2009.00548.x

Ranjan, Alka. y George, Tony. (2014). Evaluating social desirability bias in assessment ProQuest. 5(8), 948-950. Recuperado de http://search.proquest.com.proxys.mercer.edu/docview/1619362207/1FCACABF311D4159PQ/14?accountid=12381

Sandoval-Poveda, Alicia., González-Rojas, Viviana. y Madriz-Bermúdez, Linda. (2020). Retos y oportunidades: teatro como estrategia de mediación pedagógica para el desarrollo de habilidades sociales. Innovaciones Educativas, 22(32), 65-77. https://doi.org/10.22458/ie.v22i32.2821

Scott-Barrett, Juliete., Cebula, Katie., and Florian, Lani. (2018). Listening to young people with autism: learning from researcher experiences. International Journal of Research \& Method in Education, 1-22. doi:10.1080/1743727x.2018.1462791

Shaughnessy, Nicola. (2016). Curious Incidents: Pretend Play, Presence, and Performance Pedagies in Encounters with Autism. En P. Smagorinsky (Ed.), Creativity and Community among Autism-Spectrum Youth (pp.187-216). New York: Palgrave Macmillan.

Sherratt, Dave., y Peter, Melanie. (2002). Developing play and drama in children with autistic spectrum disorders. New York: David Fulton. 
Smagorinsky, Peter. (2016) Creativity and Community among Autism-Spectrum Youth. New York: Palgrave Macmillan.

Stack, Erin., y McDonald, Katherine. (2014). Nothing about us without us: Does action research in developmental disabilities research measure up? Journal of Policy and Practice in Intellectual Disabilities, 11(2), 83-91. doi:10.1111/jppi.12074

Susinos, Teresa., y Parrilla, Ángeles. (2013). Investigación Inclusiva en Tiempos Difíciles. Certezas provisionales y debates pendientes. Revista Iberoamericana sobre Calidad, Eficacia y Cambio en Educación, 11(2), 87-98. Recuperado de https://revistas.uam.es/reice/article/view/2898

Thergaonkar, Neerja., y Daniel, Deborah. (2019). Effect of arts based therapy on functionality of children with intellectual disability. Journal of Indian Association for Child \& Adolescent Mental Health, 15(2), 55-71.

Tesfaye, Rackeb., Courchesne, Valerie., Yusuf, A. Afiqah., Savion-Lemieux, Tal., Singh, Ilina., Shikako-Thomas, Keiko, ... Elsabbagh, Mayada. (2019). Assuming ability of youth with autism: Synthesis of methods capturing the first-person perspectives of children and youth with disabilities. Autism, 23(8) 1-15. doi:10.1177/1362361319831487

Toledo, Alison. (2020). Beneficios del teatro pedagógico en la comunicación en niños con Síndrome de Down (Tesis de grado en Maestro de Educación Primaria). Recuperado de https://riull.ull.es/xmlui/handle/915/20722

Trowsdale, Jo., y Hayhow, Rrichard. (2013). Can mimetics, a theatre-based practice, open possibilities for young people with learning disabilities? A capability approach. British Journal of Special Education, 40(2), 72-79. doi:10.1111/1467-8578.12019

Vargas-Pineda, Diana., y López-Hernández, Orlando. (2020). Experiencias de artistas con discapacidad frente a la promoción de la inclusión social. Arte, Individuo y Sociedad, 32(1), 31-44. doi:10.5209/ARIS.60622

Victoria, Jorge. (2013). El modelo social de la discapacidad: una cuestión de derechos humanos. Boletín Mexicano de Derecho Comparado, 46(138), 1093-1109. Recuperado de http://www.scielo.org.mx/pdf/bmdc/v46n138/v46n138a8.pdf

Zyga, Olena., Russ, Sandra., Meeker, Heather., y Kirk, Jodi. (2017). A preliminary investigation of a school-based musical theater intervention program for children with intellectual disabilities. Journal of Intellectual Disabilities, 22(3), 262278. doi:10.1177/1744629517699334 
Revista indizada en

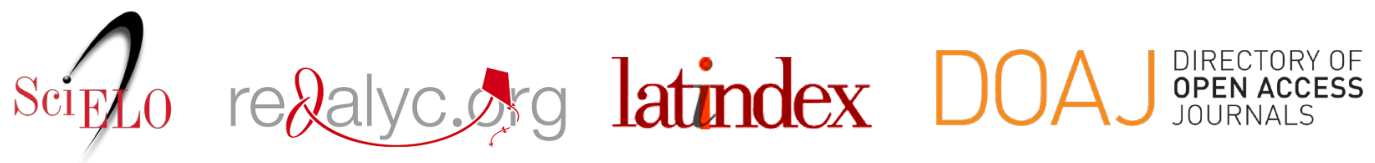

Distribuida en las bases de datos:

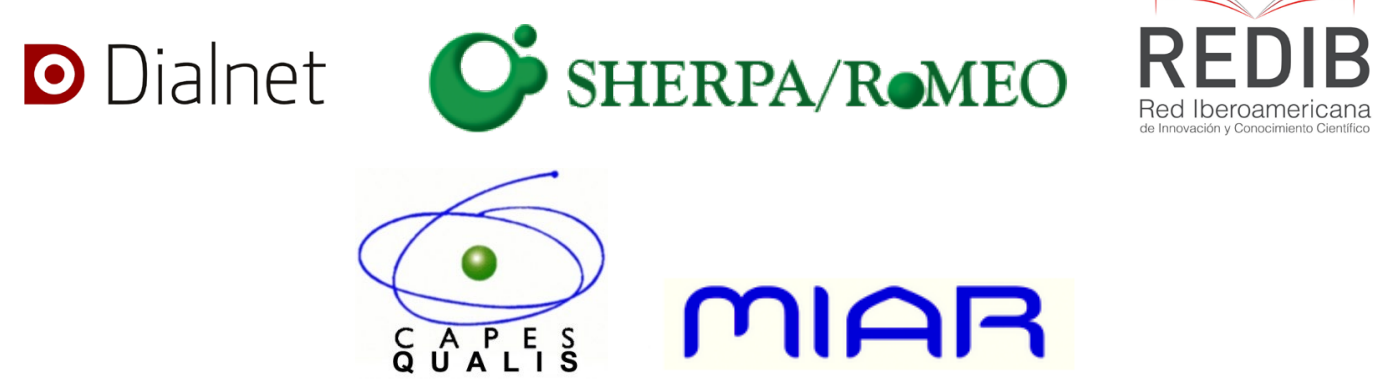

